\title{
Impact of lockdown during COVID-19 emergency on glucose metrics of children and adolescents with type 1 diabetes in Piedmont, Italy
}

\author{
Davide Tinti · Silvia Savastio ${ }^{2} \cdot$ Caterina Grosso ${ }^{3} \cdot$ Valeria De Donno ${ }^{4} \cdot$ Michela Trada $\cdot$ Martina Nugnes ${ }^{2} \cdot$ \\ Enrica Bertelli ${ }^{3}$. Luisa Franceschi ${ }^{4}$ Martina Marchisio ${ }^{1}$. Erica Pozzi ${ }^{2}$ - Eleonora Tappi ${ }^{4}$. Enrico Felici ${ }^{3}$. \\ Luisa De Sanctis ${ }^{1} \cdot$ Ivana Rabbone ${ }^{2}$ (i)
}

Received: 18 January 2021 / Accepted: 3 March 2021 / Published online: 15 March 2021

(c) Springer-Verlag Italia S.r.l., part of Springer Nature 2021

Keywords COVID-19 - Glycemic control · Children · Adolescents · Time in range · Type 1 diabetes

\section{Introduction}

From late December 2019, Severe Acute Respiratory Syndrome Coronavirus 2 has spread throughout the world, with Italy recording the first outbreak in Europe. To limit contagion, the Italian government implemented a national lockdown, which started in Piedmont (one of the most affected area in Italy) on February 23rd with schools. Complete lockdown, with sports and educational activities, started thereafter (9th of March 2020). Remote school lessons forced children and adolescents to stay home, with an overturned daily routine. Visits and scheduled checkups were canceled, further impacting physical and psychological health, with a potential negative outcome on glycemic control and acute complications. In fact, from early reports, risk of Coronavirus Disease-19 (COVID-19) progression to severe disease was higher among adult with diabetes [1]. However, children and adolescent with type 1 diabetes mellitus (T1DM) do not

Managed by Antonio Secchi .

Ivana Rabbone

ivana.rabbone@uniupo.it

1 Center of Pediatric Diabetology, AOU Città Della Salute E Della Scienza, Piazza Polonia, 94, Turin, Italy

2 Division of Pediatrics, Department of Health Sciences, University of Piemonte Orientale, V. Solaroli 17, 28100 Novara, Italy

3 Pediatric and Pediatric Emergency Unit, The Children Hospital, AO SS Antonio E Biagio E C. Arrigo, Via Venezia, 16, Alessandria, Italy

4 Department of Pediatrics, Cuneo Hospital, Via Antonio Carle, 5, Cuneo, Italy show higher morbidity and mortality related to COVID-19 [2].

We aimed to determine the impact of lockdown on patients with T1DM using a continuous glucose monitoring (CGM), and the effect of remote consultations on glucose metrics.

\section{Subjects and Methods}

We randomly selected children and adolescents ( $0-18$ years) without comorbidities (such as celiac, thyroiditis, or other autoimmune diseases) with T1DM from at least 1 year, among patients followed in the four Centers of the Piedmont Pediatric Diabetes Network. Patients were randomly selected among those who had been using a CGM (Dexcom G5/G6) for at least 6 months. Selected patients were invited to participate through e-mail and were enrolled if informed consent was signed. Ethics approval was provided by a central institutional review board.

Time spent in range (TIR), $(70-180 \mathrm{mg} / \mathrm{dL}$, $3.9-10 \mathrm{mmol} / \mathrm{L})$, below range $(\mathrm{TBR})(<70 \mathrm{mg} / \mathrm{dL}$, $<3.9 \mathrm{mmol} / \mathrm{L})$, above range (TAR) $(>180 \mathrm{mg} / \mathrm{dL},>$ $10 \mathrm{mmol} / \mathrm{L}$ ), as well as coefficient of variation (CV), sensor use, and glucose management index (GMI), were extracted during 90 days of lockdown (24th February 2020-24th May 2020) and compared with those 90 days before (25th November 2019-23rd February 2020). HbA1c values, measured at the last available visit before lockdown, were collected. Patients reported physical activity (hours per week) and total daily insulin dose (TDD) in the same study period, both before and during lockdown. Every patient was offered a remote visit and, if interested, was called back by the physician and obtained remote consultation via telephone. 
Primary end point was to evaluate changes in CGM metrics during lockdown in a cohort of children and adolescent with T1DM, compared to the previous period. Secondary end point was to evaluate remote consultation impact on glycemic control during lockdown. Demographic variables were summarized by mean \pm standard deviation $(\mathrm{SD})$ or percentage (\%), and they were compared using a t test or a paired-sample $t$ test for continuous variables, with an alpha value of 0.05 . A multivariate regression was conducted to analyze relationship between TIR during lockdown with analyzed variables. Analyses were performed using SPSS (B) statistical software (24th edition, IBM).

\section{Results}

Of the patients invited to participate, 66 were enrolled. Mean age was $11.6 \pm 4.5$ years, subdivided in three age groups ( 8 children $0-6$ years, 22 children $6-12$ years, and 36 adolescents $12-18$ years). The mean value of $\mathrm{HbA1c}$ was 7.2 $\pm 0.8 \%(53 \pm 9 \mathrm{mmol} / \mathrm{L})$ and BMI Z-score was $0.386 \pm$ 1.13. Fifty-eight (88\%) were carbohydrate counters, while $36(55 \%)$ used an insulin pump (Table 1). No participant reported COVID-19 infection, diabetic ketoacidosis, or severe hypoglycemia during the study.

Table 1 Characteristics of the study participants. Data are expressed as number \pm SD unless otherwise indicated. BMI: Body Mass Index

\begin{tabular}{ll}
\hline & $n=66$ \\
\hline Age (years) & $11.6 \pm 4.5$ \\
0 to $<6, n(\%)$ & $8(12)$ \\
6 to $<12, n(\%)$ & $22(33)$ \\
12 to $<18, n(\%)$ & $36(55)$ \\
Sex, $n$ of males $(\%)$ & $46(65)$ \\
BMI $\left(\mathrm{kg} / \mathrm{m}^{2}\right)$ & $18.6 \pm 2.8$ \\
BMI Z-score & $0.386 \pm 1.13$ \\
Waist circumference $(\mathrm{cm})$ & $65 \pm 8$ \\
Hip circumference $(\mathrm{cm})$ & $75 \pm 12$ \\
Disease duration, (years) & $4.5 \pm 3$ \\
1 to $<2, n(\%)$ & $13(20)$ \\
2 to $<5, n(\%)$ & $28(43)$ \\
$\geq 5, n(\%)$ & $24(37)$ \\
HbA1c $(\%)$ & $7.2 \pm 0.8$ \\
HbA1c (mmol/mol) & $55 \pm 9$ \\
$<7 \%(53 \mathrm{mmol} / \mathrm{mol}), n, \%$ & $25(38)$ \\
$7 \%$ to $<7.5 \%(53$ to $<58 \mathrm{mmol} / \mathrm{mol}), n(\%)$ & $18(27)$ \\
$7.5 \%$ to $<8 \%(58$ to $<64 \mathrm{mmol} / \mathrm{mol}), n(\%)$ & $14(21)$ \\
$8 \%$ to $<10 \%(64$ to $<86 \mathrm{mmol} / \mathrm{mol}), n(\%)$ & $9(14)$ \\
Carbohydrate counters, $n(\%)$ & $58(88 \%)$ \\
Type of therapy, $n$ of $\mathrm{CSII}(\%)$ & $36(55 \%)$ \\
\hline
\end{tabular}

Before lockdown, participants showed a mean glucose of $168 \pm 61 \mathrm{mg} / \mathrm{dL}(9.3 \pm 3.4 \mathrm{mmol} / \mathrm{L})$, while during lockdown was $165 \pm 58 \mathrm{mg} / \mathrm{dL}(9.2 \pm 3.2 \mathrm{mmol} / \mathrm{L})(P<0.05)$. TIR increased from $59.7 \pm 13 \%$ to $62.5 \pm 14 \%(P=0.001)$, while TAR decreased from $37.8 \pm 14 \%$ to $35.2 \pm 15 \%(P$ $=0.004)$, as shown in Fig. 1. No significant differences were detected for TBR (from $2.5 \pm 2.3 \%$ to $2.3 \pm 2.5 \%, P=$ 0.177 ) and GMI (from $7.5 \pm 0.9 \%$ to $7.4 \pm 0.8 \%, P=0.05$ ). $\mathrm{CV}$ decreased from $36 \pm 5 \%$ to $35 \pm 5 \%(P=0.003)$. Physical activity spent per week reduced from $6.1 \pm 3.3 \mathrm{~h}$ to 2.7 $\pm 3.1 \mathrm{~h}(P<0.001)$, while TDD increased from $0.79 \pm 0.25$ $\mathrm{UI} / \mathrm{kg} /$ day to $0.87 \pm 0.31 \mathrm{UI} / \mathrm{kg} /$ day $(P=0.004)$. CGM use increased from $87 \pm 17$ to $92 \pm 10 \%$ of time $(P=0.006)$. Multivariate regression about TIR during lockdown did not observe associations, except for HbA1c before lockdown ( $\beta$ $-0.696)$.

We did not observe significant differences in TIR (61.5 $\pm 15 \%$ versus $63.7 \pm 13 \%)$, TBR $(2.1 \pm 2.4 \%$ versus $2.5 \pm$ $2.6 \%)$, TAR $(36.4 \pm 16 \%$ versus $33.8 \pm 13 \%)$, and CV ( $35 \pm$ 5 versus $35 \pm 5 \%)$, as well as TDD $(0.85 \pm 0.27$ versus 0.89 $\pm 0.37 \mathrm{UI} / \mathrm{kg} / \mathrm{day})$ in patients who received remote consultations $(n=40)$ during lockdown.
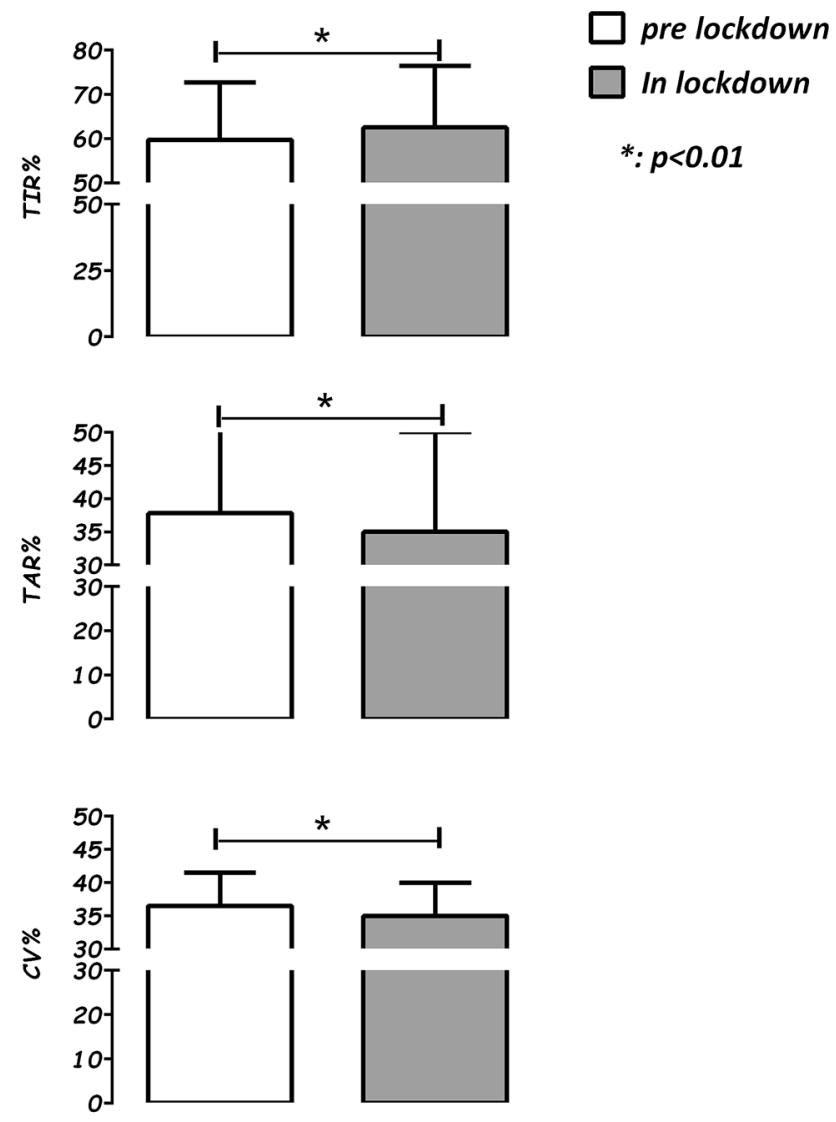

Fig. 1 Plots of time spent in range (TIR), above range (TAR) and coefficient of variation (CV) before (in white) and during lockdown (in grey) 


\section{Discussion}

Our study population was in good metabolic control, with roughly half of them using an insulin pump. Before lockdown, patients demonstrated a mean TIR of $59.7 \%$, mean TBR of $2.5 \%$, and mean TAR of $37.8 \%$, approaching International Recommendation targets and similar to those observed in Italian children [3].

Enrolled subjects were physically active, with a mean of $6.1 \mathrm{~h}$ per week, which dropped during lockdown $(-3.4 \mathrm{~h} /$ week), probably leading to a higher TDD (around $0.1 \mathrm{UI} / \mathrm{kg}$ / day). Despite this, patients did not demonstrate a worsening in CGM metrics, with a mild improvement of TIR $(+2.8 \%)$, TAR (-2.6\%), and CV (-1\%) instead.

A study has been recently published in a group of T1DM pediatric subjects, evaluating glucose metrics in a shorter period of time ( 2 weeks), with no deterioration or improvement of the glycemic balance [4].

We conclude that seeing the lockdown period as a whole, children and adolescents (and their caregivers) using a CGM were able to adjust insulin therapy despite less physical activity and a different lifestyle.

On the other hand, the improvement in glucose metrics could be due to less stress related to school and diabetes management, as some authors also suggested [5]. Furthermore, in our observation, remote consultation was requested by $60 \%$ of patients but did not impact significantly glucose control.

\section{Conclusion}

Despite less physical activity, CGM metrics of children and adolescents with T1DM in the Piedmont Region did not worsen but, instead, slightly improved during lockdown due to COVID-19 emergency. Special attention should be paid to diabetes management at school for children and adolescent with T1DM.

Funding This research did not receive any specific grant from funding agencies in the public, commercial, or not-for-profit sectors.

\section{Declarations}

Conflict of interest The authors declare that they have no conflict of interest.

Informed Consent Informed consent was obtained from every subject participating the study.

Human and Animal Rights All procedures performed in studies involving human participants were in accordance with the ethical standards of the institutional and/or national research committee and with the 1964 Helsinki declaration and its later amendments or comparable ethical standards.

\section{References}

1. Singh AK, Gupta R, Ghosh A, Misra A (2020) Diabetes in COVID-19: Prevalence, pathophysiology, prognosis and practical considerations. Diabetes metabolic syndrome 14(4):303-310. https://doi.org/10.1016/j.dsx.2020.04.004

2. Pitocco D, Tartaglione L, Viti L et al. (2020) Lack of type 1 diabetes involvement in SARS-COV-2 population: Only a particular coincidence? Diabetes Res Clin Pract 164:108220. https://doi.org/ 10.1016/j.diabres.2020.108220

3. Cherubini V, Bonfanti R, Casertano A et al. (2020) Time in range in children with type 1 diabetes using treatment strategies based on nonautomated insulin delivery systems in the real world. Diabetes Technol Ther. https://doi.org/10.1089/dia.2020.0031

4. Brener A, Mazor-Aronovitch K, Rachmiel M et al. (2020) Lessons learned from the continuous glucose monitoring metrics in pediatric patients with type 1 diabetes under COVID-19 lockdown. Acta Diabetol 57(12):1511-1517. https://doi.org/10.1007/ s00592-020-01596-4

5. Schiaffini R, Barbetti F, Rapini N et al. (2020) School and preschool children with type 1 diabetes during Covid-19 quarantine: The synergic effect of parental care and technology. Diabetes Res Clin Pract 166:108302. https://doi.org/10.1016/j.diabres.2020. 108302

Publisher's Note Springer Nature remains neutral with regard to jurisdictional claims in published maps and institutional affiliations. 